\title{
Avaliação da experiência do telemonitoramento de enfermagem por mulheres com excesso de peso
}

\author{
Assessment of the experience of nursing telemonitoring by overweight women \\ Evaluación de la experiencia de la telemonitorización de enfermería por mujeres con exceso de peso
}

Catia Suely Palmeira ${ }^{1}$ (D)

Giulia Araújo Ramos ${ }^{1}$ (1)

Fernanda Carneiro Mussi ${ }^{2}$ (D)

1. Escola Bahiana de Medicina e Saúde

Pública. Salvador, BA, Brasil.

2. Universidade Federal da Bahia, Escola de

Enfermagem. Salvador, BA, Brasil.
Autor correspondente:

Catia Suely Palmeira

E-mail: catia_palmeira@yahoo.com.br

Recebido em 23/03/2020.

Aprovado em 30/06/2020.

DOI:https://doi.org/10.1590/2177-9465-EAN-2020-0090

\section{Resumo}

Objetivo: Avaliar a experiência do monitoramento remoto de enfermagem na perspectiva de mulheres com excesso de peso. Método: Estudo descritivo, de abordagem qualitativa, realizado em ambulatório de referência em obesidade em Salvador-Bahia, com 42 mulheres com excesso de peso, as quais haviam participado do grupo intervenção de um ensaio clínico randomizado. Realizaram-se entrevistas semiestruturadas entre janeiro a março de 2017 e os dados foram analisados através da técnica de análise de conteúdo temática. Resultados: Dos depoimentos emergiu a categoria central "Aumentando a consciência do cuidado de si", a qual foi representada por três categorias temáticas: Vivenciando um feedback frequente e interativo com a enfermeira para o controle de peso, Melhorando o cuidado de si e Sentindo satisfação com os resultados alcançados. Conclusão e implicações para a prática: As participantes vivenciaram e reconheceram a melhora do conhecimento para o controle do peso e mudanças positivas nos modos de viver ou conviver com o excesso de peso. Os resultados evidenciaram que atividades educativas por meio da telenfermagem, numa perspectiva dialógica, contribuem para potencializar o autocuidado.

Palavras-chave: Obesidade; Saúde da Mulher; Telemonitoramento; Perda de peso; Educação em Saúde.

\section{Abstract}

Objective: To evaluate the experience of remote nursing monitoring from the perspective of overweight women. Method: Descriptive qualitative approach study, carried out in a reference outpatient clinic in obesity in Salvador-Bahia, with 42 overweight women, who had participated in the intervention group of a randomized clinical trial. Semi-structured interviews were conducted between January and March 2017. The data were analyzed using the thematic content analysis technique. Semi-structured interviews were conducted between January and March 2017, with the data having been analyzed through thematic content analysis. Results: From their statements the central category "Increasing awareness for self-care" was identified, which was represented by three thematic categories: Experiencing a frequent and interactive feedback with the nurse for weight control, Improving self-care and Feeling satisfaction with the results obtained. Conclusion and implications for practice: The participants experienced and recognized improved knowledge for weight control and positive changes in ways of living or living with excess weight. The results evidenced that educational activities, through telenursing, in a dialogical perspective, contribute towards enhancing self-care.

Keywords: Obesity; Women's Health; Telemonitoring; Weight loss; Health Education.

\section{REsumen}

Objetivo: Evaluar la experiencia de la monitorización remota de enfermería en la perspectiva de las mujeres con exceso de peso. Método Estudio descriptivo, de enfoque cualitativo, realizado en ambulatorio de referencia en obesidad en Salvador-Bahia, con 42 mujeres con exceso de peso, las cuales habían participado del grupo de intervención de un ensayo clínico aleatorio. Se realizaron entrevistas semiestructuradas entre enero y marzo de 2017 y los datos fueron analizados mediante la técnica de análisis de contenido temático. Resultados: De los testimonios surgió la categoría central "Aumentando la conciencia sobre el autocuidado", la cual estaba representada por tres categorías temáticas: Vivenciando un feedback frecuente e interactivo con la enfermera para el control del peso, Mejorando el autocuidado y Sintiendo satisfacción con los resultados logrados. Conclusión e implicaciones para la práctica: Las participantes vivenciaron y reconocieron la mejora del conocimiento para el control de peso y cambios positivos en los modos de vivir o convivir con exceso de peso. Los resultados evidenciaron que actividades educativas por medio de la teleenfermería, en una perspectiva dialógica, contribuyen para potencializar el autocuidado.

Palabras clave: Obesidad; Salud de la Mujer; Telemonitorización; Pérdida de peso; Educación en Salud. 


\section{INTRODUÇÃO}

Tem havido, atualmente, um aumento significativo da obesidade em diversas populações do mundo e a sua prevalência mais do que duplicou entre 1980 e $2014 .{ }^{1}$ A obesidade tem uma etiologia complexa e multifatorial, sendo causada por um desequilíbrio energético entre as calorias consumidas e gastas. ${ }^{1}$ Os fatores que contribuem para o acúmulo de peso variam de pessoa para pessoa e sofrem influências da genética e do estilo de vida. ${ }^{2}$

No mundo moderno, além do aumento no consumo dos alimentos industrializados, que são ricos em gordura, de fácil acesso e grande praticidade, também vem ocorrendo um aumento na redução da prática de atividade física. Esta se deve à influência da natureza sedentária de novas formas de trabalho, mudanças em meios de transporte e aumento da urbanização. ${ }^{1}$

A obesidade é um fator de risco para muitas doenças crônicas não transmissíveis (DCNT), como as doenças cardiovasculares, diabetes mellitus tipo II, distúrbios do músculo esquelético e alguns tipos de câncer. Existem evidências que quanto maior o IMC, maior é o risco para as DCNT e maior a taxa de mortalidade,,$^{1,3}$ além de custos substanciais com a saúde e a sobrecarga para os serviços de saúde. ${ }^{4}$

Dentre as várias implicações da obesidade, destacam-se as psicossociais que geram sofrimento, preconceito, discriminação, não aceitação da imagem corporal, baixa autoestima, isolomento social e depresssão. Existe também o risco de perda permanente do trabalho devido a aposentadoria por invalidez e morte prematura ou perda temporária em razão de licença por doença e redução da produtividade..$^{4,5}$

O tratamento da obesidade é complexo, multidisciplinar e envolve mudanças no estilo de vida. Estas mudanças se baseiam em medidas relacionadas aos hábitos alimentares saudáveis e a prática regular de atividade físca. O sucesso, em longo prazo, depende de cuidados constantes na adequação destas medidas, além de outros fatores, como apoio social, familiar e automonitorização. ${ }^{3} \mathrm{O}$ tratamento deve considerar, além do controle do peso em longo prazo, os efeitos benéficos sobre as doenças associadas, como diabetes mellitus tipo 2, hipertensão arterial e dislipidemia. ${ }^{2}$ Devido ao aumento crescente da obesidade no mundo, é premente o uso de diferentes estratégias direcionadas ao cuidado da pessoa obesa.

Considerando que a obesidade é uma doença crônica que tende a recorrer após a perda de peso, as pessoas obesas precisam de apoio e acompanhamento de profissionais de saúde em futuro distante. A sua abordagem deve ser individualizada e ter como meta melhorar o conhecimento sobre o problema e a motivação para agir contra os fatores obesogênicos ambientais. ${ }^{3}$

Dentre as práticas de cuidado, destaca-se a educação em saúde como uma ferramenta valiosa para auxiliar pessoas com excesso de peso a desenvolver a consciência sobre a doença e a enfrentar as barreiras do controle do peso. ${ }^{6} \mathrm{O}$ (a) enfermeiro (a) por meio de práticas educativas desempenha um importante trabalho no empoderamento do usuário para a promoção da saúde. Estas práticas contribuem, ainda, para o cuidado humanizado focado em uma relação dialógica entre quem cuida e quem é cuidado e no compartilhamento e valorização de saberes, experiências e decisões. ${ }^{7}$

A criação de novas abordagens que auxiliem a perda e a manutenção do peso perdido e a melhora das condições gerais de saúde deve ser meta e responsabilidade da equipe e dos gestores de saúde, assim como das autoridades públicas. $\mathrm{O}$ monitoramento remoto pode ser considerado uma estratégia inovadora e viável, sendo conhecido como uma ferramenta que envolve várias tecnologias de informação e comunicação (TICs) que favorecem a acessibilidade do usuário ao profissional de saúde. ${ }^{8}$

Atualmente, as TICs têm sido reconhecidas nos programas de saúde e utilizadas na atenção a pessoas com doenças crônicas. O uso desta tecnologia contribui para a prevenção, diagnóstico, monitoramento e tratamento da doença, permitindo a comunicação entre as pessoas, a reunião de informações e interação com os serviços a distância, mais rapidamente, e sem limitação de tempo e lugar. Para os profissionais de saúde há o aumento da comunicação e apoio, possibilitando o diagnóstico, intervenções precoces e economia do tempo de trabalho. Em contrapartida, há redução da taxa de internações hospitalares e consultas ambulatoriais para o sistema de saúde. ${ }^{9}$ Por fim, a população é beneficiada com o empoderamento, a diminuição da ansiedade e a melhora no gerenciamento da doença e do tratamento.

O telemonitoraomento tem sido cada vez mais adotado para ajudar as pessoas a gerenciar melhor os cuidados em saúde e tratar a doença em fase crônica em casa, como o infarto do miocárdio, a diabetes mellitus tipo II, a insuficiência renal e as doenças pulmonares. ${ }^{10,11}$ Entretanto, ainda há escassez de publicações sobre o uso desta tecnologia no cuidado relacionado ao controle do excesso de peso. ${ }^{12}$

No Brasil, por meio de ampla busca nas principais bases de dados e bibliotecas online Scientific Electronic Library Online (SciELO), Biblioteca Virtual em Saúde (BVS) Latin America and the Caribbean Literature on Health and Science (Lilacs) e Medical Literature Analysis and Retrieval System Online (MEDLINE), o único estudo identificado sobre monitoramento remoto de enfermagem com mulheres com excesso de peso foi desenvolvido por Palmeira et al. ${ }^{13}$ Desse modo, torna-se relevante conhecer e divulgar a avaliação do monitoramento realizada pelas mulheres, participantes do estudo, ${ }^{13}$ visando oferecer à comunidade acadêmica e aos profissionais da saúde outras evidências científicas sobre a ferramenta utilizada.

Diante dessas considerações, este estudo objetivou avaliar a experiência do monitoramento remoto de enfermagem para mulheres com excesso de peso.

\section{MÉTODOS}

Trata-se de um estudo descritivo, de abordagem qualitativa, realizado em um ambulatório de referência em obesidade, de uma instituição de ensino superior privada, na cidade de Salvador-BA.

As participantes foram mulheres com excesso de peso, acompanhadas no lócus de estudo pelo "Projeto de Estudo do 
Excesso de Peso e Doença Cardiometabólica" (PEPE) e que participaram de um projeto de pesquisa matriz, com desenho de ensaio clínico randomizado. A população desse projeto matriz foi formada por 101 mulheres alocadas randomicamente em dois grupos, sendo 51 mulheres no grupo de intervenção (GI) e 50 mulheres no grupo-controle (GC).

Os critérios de inclusão das mulheres para o ensaio clínico foram: ter excesso de peso (IMC $\geq 25(\mathrm{~kg} / \mathrm{m} 2)$, ter idade superior a 18 e inferior a 60 anos, ter comparecido, no mínimo, a uma consulta nos últimos doze meses e ter telefone fixo e/ou celular. Foram excluídas àquelas com déficit cognitivo ou transtornos psiquiátricos graves que as impediriam de responder formulários, que estivessem em uso de drogas para perda de peso e àquelas submetidas à cirurgia bariátrica.

O critério de elegibilidade das mulheres para o presente estudo foi ter pertencido ao GI do ensaio clínico e ter participado no monitoramento remoto realizado pelo projeto matriz. O GI foi, inicialmente, constituído por 51 mulheres, mas, durante o desenvolvimento do monitoramento remoto, ocorreram nove perdas, sendo seis no inicio da intervenção e três na última semana. Desta forma, a população total do presente estudo foi de 42 mulheres.

A intervenção realizada por monitoramento remoto, objeto de avaliação desta pesquisa, teve duração de três meses e se constituiu de atividades educativas sobre o excesso de peso, realizadas por contato telefônico. As ligações ocorriam uma vez por semana e obedeciam a um roteiro temático para a promoção da saúde, prevenção e controle do excesso de peso e a um guia de orientação telefônica para a abordagem das mulheres.

A coleta de dados ocorreu após a última semana da intervenção e foi realizada no período de janeiro a março de 2017 por meio de uma entrevista semiestruturada, contendo duas perguntas abertas: "o que a senhora achou do projeto do monitoramento feito por meio de ligações telefônicas?" e o que a senhora achou das orientações dadas?

As entrevistas foram realizadas pelo telefone, gravadas por um aplicativo para smartphone denominado Automatic Call Recoder e depois transcritas na íntegra. Catorze entrevistas foram repetidas, presencialmente, com o objetivo de aprofundar e melhor compreender o conteúdo das falas das mulheres, respeitando-se a disponibilidade da presença da participante para realizar a coleta dos dados pós-intervenção. Para preservar o anonimato foi atribuída a letra "E" para identificação da mulher entrevistada, as quais receberam o número de 1 a 42 .

$\mathrm{Na}$ análise de dados, empregou-se a análise de conteúdo temática que abrangeu as seguintes fases: pré-análise, exploração do material, tratamentos dos resultados e interpretação. ${ }^{11,14}$ Após a transcrição das gravações telefônicas e das entrevistas presenciais, procedeu-se à leitura minuciosa de todos os depoimentos com o objetivo de identificar os núcleos de sentido. Posteriormente, buscou-se refletir com maior profundidade sobre o conteúdo desses núcleos, apreendê-los em sua essência, para então realizar o seu agrupamento por similaridades e diferenças, dando origem às categorias e subcategorias. Os resultados foram interpretados, estabelecendo-se articulação com a literatura e com o objetivo do estudo.

O projeto matriz foi aprovado pelo Comitê de Ética em Pesquisa da Escola de Enfermagem da UFBA, CAAE: 43665115.6 .0000 e seguiu as exigências da resolução 466/2012 do Conselho Nacional de Saúde, que trata da pesquisa em seres humano. Todas as participantes leram e assinaram o Termo de Consentimento Livre e Esclarecido.

\section{RESULTADOS}

A categoria central que representa a avaliação do monitoramento remoto de enfermagem pelas mulheres com excesso de peso o "Aumentando a consciência do cuidado de si", a qual se expressa em três categorias: Vivenciando um feedback frequente e interativo com a enfermeira para o controle de peso, Melhorando o cuidado de si, Sentindo satisfação com os resultados alcançados, conforme mostra a Figura 1.

A categoria central "Aumentando a consciência do cuidado de si" expressou que o monitoramento remoto de enfermagem para mulheres com excesso de peso se constituiu em uma intervenção interativa com a enfermeira que contribuiu para fortalecer conhecimentos, ocuparem-se do cuidado consigo mesmo, adaptarem-se a novos hábitos, manterem-se na linha, lograrem mudanças de comportamento e sentirem-se valorizadas.

$\mathrm{O}$ aumento da consciência do cuidado de si foi resultante do processo de interação experienciado durante o período de monitoramento, conforme expressam três categorias e respectivas subcategorias.

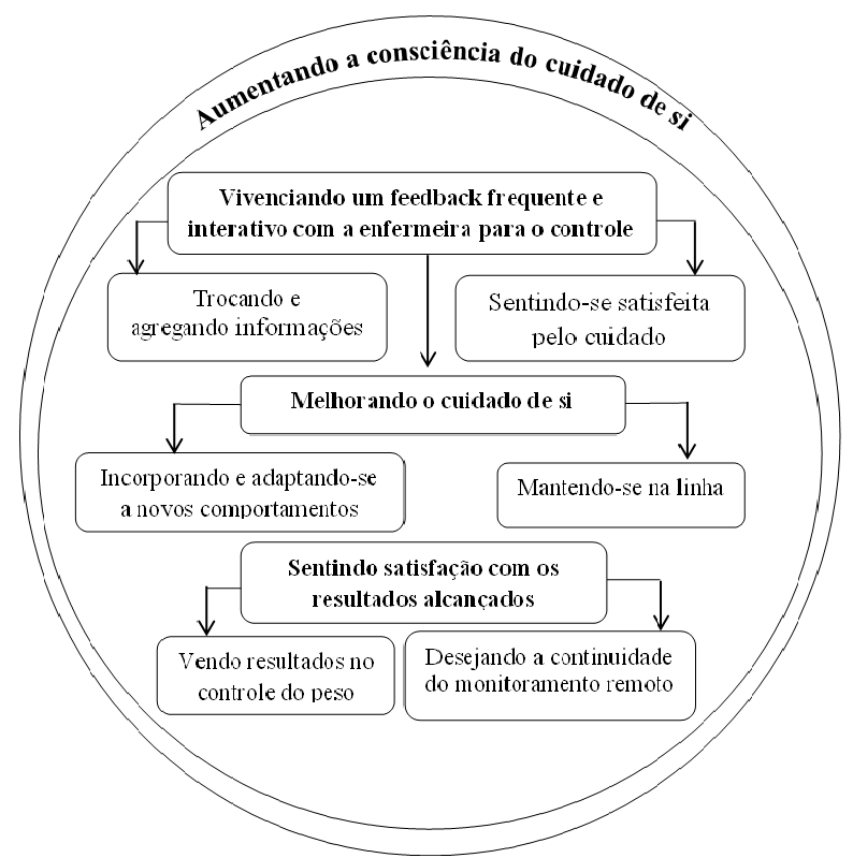

Figura 1 - Diagrama representativo da avaliação do telemonitoramento de enfermagem por mulheres com excesso de peso

Fonte: Elaboração própria 


\section{Categoria 1: Vivenciando um feedback frequente e interativo com a enfermeira para o controle de peso.}

Essa categoria e suas duas subcategorias retrataram que o monitoramento promoveu um encontro entre a enfermeira e as mulheres, momentos de trocas intersubjetivas que permitiam compartilhar e agregar conhecimentos e sentir satisfação pelo cuidado compartilhado.

Trocando e agregando conhecimento revelou que o monitoramento remoto foi considerado pelas mulheres uma estratégia valiosa para esclarecer dúvidas sobre o excesso de peso e seu controle, conforme ilustram os depoimentos:

\section{Ajudou sim, perfeitamente, étanto que agora tirou algumas dúvidas (E20). \\ Não ficou dúvida sobre alimentação, sobre o que deve comer e sobre o que deve beber. No meu caso, eu já bebia muita água, tinha consciência de que tinha que beber água já que tomo muito medicamento, tudo foi bem vindo, foi bem aceito (E31).}

Além disso, expressou a avaliação positiva dessa intervenção, ao reforçar e relembrar conhecimentos prévios.

Foi ótimo vocês estarem ligando e dando orientação. Apesar de que eu já estava sabendo, mas orientações sempre ajudam e são bem vindas (E3).

Achei ótimo, ajuda estar lembrando que você tem que cumprir as metas e lembrar de algumas práticas que tem que ser feitas (E25).

Achei ótima, foi muito boa, lembrou a gente do que podia continuar fazendo, e eu tentei fazer o máximo que eu pude dentro do meu limite (E26).

Foi ótimo, porque é um reforço e um incentivo para que a gente tenha consciência da nossa saúde e possa preservar e cuida (E31).

No processo de interação telefônica as mulheres também descobriram novas informações:

Eu achei maravilhoso, amei, É um incentivo muito grande, pois a gente vai descobrindo mais coisas que estão faltando. Eu gostei muito, foi muito satisfatório estar recebendo as ligações de vocês (E5).

Meninas vocês orientaram bem a gente. Sobre a alimentação a gente sabe quase de tudo, mas sempre vão aparecendo coisas novas, vai renovando. (E1).

Eu gostei das ligações. Deram uma orientação melhor para quem não tem tanto conhecimento (E24).

Sentindo-se satisfeita pelo cuidado compartilhado foi outra subcategoria que revelou que as mulheres sentiram-se motivadas, incentivadas, com forças para seguir o tratamento e bem avaliadas no feedback interativo com a enfermeira. As orientações continuadas serviram de estímulo e força, inclusive, em momentos de desmotivação.

Eu gostei, foi muito bom para gente, porque incentivou, a gente às vezes precisa de incentivo mesmo. O que eu achei muito importante no monitoramento, primeiramente, foi para nos dar força. Porque a gente precisa de força para lutar (E28).

Achei ótimo. Para mim foi perfeito, foi muito bom, gostei muito, tinha algumas situações ou horas em que eu estava desmotivada e aí a ligação vinha e fazia com que eu me animasse novamente (E4).

Eu gostei muito, porque dá uma força a mais pra gente, como levantar mais a autoestima, gostei muito (E38).

Muitas mulheres sentiram-se únicas e valorizadas pelo cuidado oferecido, ao ter alguém que se preocupava com seu estado de saúde.

Eu gostei muito porque é difícil você ter médico que fica ligando para o paciente, para se preocupar com ela. Então, estou me sentindo a própria. Parece que é tudo particular. Vocês são especiais para gente, estava dizendo a uma amiga minha, tomara que não acabe nunca porque é muito bom, depois que eu estou nesse projeto estou me sentindo tão bem (E2).

Eu gostei, gostei muito, me sentia bem, porque toda semana tinha ligação. Você ligava para me falar e para saber como eu passei a semana e ai a gente vai falando como está passando, como está se sentindo. Eu achei maravilhoso gostei muito, muito (E1).

Incentiva a gente a fazer as atividades, a dieta. Às vezes a gente até tenta esquecer, mas quando recebe uma ligação de vocês, a gente sente alguém se preocupando, entendeu! Eu gostei muito (E35).

As mulheres também se sentiram mais bem avaliadas:

Eu achei bom, porque como é que diz, para ver se realmente a paciente está fazendo mesmo o que tem que fazer e o que é certo fazer. Eu gostei, achei legal (E9).

Para mim foi bom, vocês saberem o que a gente está comendo, o que não está, como está a saúde da gente. Então foi ótimo (E14).

\section{Categoria 2: Melhorando o cuidado de si}

Essa categoria mostrou que as mulheres vivenciaram mudanças e adaptações em hábitos de vida por meio de um feedback interativo durante o monitoramento remoto de enfermagem, 
conforme ilustram as duas subcategorias: Incorporando e adaptando-se a novos comportamentos e Mantendo-se na linha.

Incorporando e adaptando-se a novos comportamentos revelou que as mulheres adotaram novos hábitos de vida como fracionamento das refeições, prática de atividade física, redução dos excessos na dieta e do comportamento sedentário, entre outros. Além disso, experimentaram a adaptação a novos hábitos apesar das dificuldades enfrentadas. É como se fizessem um balanço nos modos de viver.

Muito bom, conscientizou-me mais. Estava até falando para as meninas que eu comecei a fazer academia, já tem quase um mês. Já estou bebendo mais água, estou fazendo mais coisas, correndo atrás (E7).

Ajudou bastante, como a caminhar mesmo e subir as escadas. Estou subindo as escadas todos os dias (E1).

Estou tentando na medida do possível fazer o máximo, ter disciplina para evitar abusos, a gente fazia coisas e agora não faz, evita, pelo menos, comer e beber certas coisas (E24)

A orientação foi muito boa, eu estou seguindo algumas. Algumas eu já consegui seguir e outras ainda estou tentando me adaptar (E4).

Ajudou sim, estou tentando na medida do possível fazer o máximo. Observar disciplina das coisas que a gente tem que fazer e os abusos que a gente fazia e agora não faz ou evita pelo menos. Eu tenho tentado fazer, seguir um pouco o projeto, mas é um pouco difícil (E24).

A subcategoria, Mantendo-se na linha, expressou que as mulheres perceberam que o monitoramento contribuiu para reforçar e assegurar o valor do seguimento das medidas de controle do peso, constituiu-se em um alerta, lembrete e estímulo para manterem-se na linha, sem que se sentissem pressionadas.

Eu acho bom, acho muito legal, isso estimula mais, ajuda a continuar porque, às vezes, a gente quer sair da linha e tendo a ligação toda semana tem um ..... do que pode e o que não pode (E6).

É muito bom, porque ai serve para ver se realmente está fazendo mesmo o que tem que fazer e o que é certo fazer. Eu gostei, achei legal e ajudou muito, lembrou que a gente tem que fazer a nossa dieta, não se esquecer de tomar o remédio (E9).

Eu gostei, porque eu achei que faz você voltar mais para linha. Quando vocês ligavam, eu acordava e voltava (E22).

Eu achei bom porque, de qualquer forma se a pessoa ficar pensando em escapulir, ai vocês ligando toda semana a gente se sente na obrigação de cumprir o nosso dever, de comer, de se alimentar da forma correta. Que você sabe que tem que beber água, comer frutas diversificadas e não uma só, se alimentar de três em três horas tudo isso foi bom porque ficou relembrando o que a gente sabe (E32).

\section{Categoria 3: Sentindo satisfação com os resultados alcançados}

Essa categoria mostrou que as mulheres expressaram satisfação com o controle ou perda do peso alcançado durante o processo de monitoramento e, assim, desejaram que o mesmo fosse permanente.

A subcategoria, Vendo resultados no controle do peso, evidenciou que algumas mulheres perderam ou não ganharam peso, o que fez com que se sentissem ajudadas e satisfeitas.

Perdipeso, perdi medida, perdi cintura, tudo Graças a Deus! Gostei muito, achei maravilhoso. Estão de parabéns (E1)

Ajudou sim com certeza, pelo menos eu mantive o peso (E26).

Ajudou sim, ajudou que eu estou conseguindo já até perder uns quilinhos (E38).

A subcategoria, Desejando a continuidade do monitoramento remoto, revelou que as mulheres lamentaram o término da intervenção e sentiram-se tristes, descontentes e saudosas.

Eu achava maravilhoso, eu nem queria que acabasse. Fiquei triste porque terminou. Na ultima semana eu fiquei triste porque demora seis meses para gente voltar ao médico. Pena que acabou, tinha que continuar (E1).

Vocês são especiais para nós, estava dizendo a uma amiga minha, tomara que não acabe nunca porque é muito bom, depois que eu estou nesse projeto estou me sentindo tão bem (E2).

Queria que continuasse com as ligações, pelo menos a gente fica na esperança de algumas coisas. No meu ver se continuasse pra mim tudo bem, para mim foi ótimo. (E20)

Desde quando soube que ia acabar senti falta e gostaria mesmo que permanecesse assim, mas infelizmente a gente sabe que não dá (E28).

Para mim o feedback foi interessante, gostei demais. Para mim foi positivo estarem me incentivando. Tomara que tenha continuação (E29).

\section{DISCUSSÃO}

Neste estudo, o monitoramento remoto de enfermagem como estratégia de educação à saúde para o controle do peso foi avaliado de forma positiva pelas participantes, principalmente, por oportunizar novas aprendizagens e capacitá-las para escolhas conscientes e adequadas. 
As participantes expressaram o surgimento de uma maior conscientização para o cuidado de si na vivência desta intervenção. A consciência, como resultado do conhecimento preexistente pode levar ao movimento, à prática de uma ação consciente $\mathrm{e}$ transformadora, ${ }^{15}$ refletida nas mudanças e adaptações de hábitos de vida feitas pelas mulheres a partir da vivência de um feedback interativo com a enfermeira. A consciência do cuidado de si pode advir do conhecimento e reflexão adquiridos em processos de educação em saúde compartilhados, os quais contribuem para despertar novas análises acerca do processo de saúde-doença e para a valorização de novos modos de viver. Intervenções educativas e mediadas por estratégias inovadoras, tal como o telemonitoramento de enfermagem, podem contribuir para a melhora do cuidado de si quando realizado de forma dialógica. ${ }^{9}$

As possibilidades de trocas de informações e o aumento do conhecimento reveladas pelas participantes vieram acompanhados de avaliações positivas e da satisfação pelo cuidado da enfermeira como evidenciou a categoria:Vivenciando um feedback frequente e interativo com a enfermeira para o controle do peso e suas subcategorias.

O conhecimento contempla um conjunto de informações que o indivíduo precisa dominar para manejar a condição de saúde ${ }^{16}$ e, no que se refere ao controle da obesidade, apesar de não ser suficiente para promover a mudança de comportamento, quando adequado, pode ser uma ferramenta valiosa para auxiliar as pessoas nas escolhas de um novo estilo de vida, e assim deve fazer parte de programa de intervenção educativa. ${ }^{17}$

Existem evidências de que o conhecimento, quando compartilhado pelos profissionais de forma dialógica e com linguagem apropriada, poderá ser assimilado pelos participantes do processo, sobretudo se seus saberes forem levados em consideração. ${ }^{18}$ Esse princípio foi adotado no monitoramento remoto de enfermagem, contribuindo para a vivência de um feedback interativo das mulheres com a enfermeira, visando o controle do peso.

No processo de educação em saúde deve ser considerado o conhecimento do indivíduo para que se possa, a partir daí, discutir erros conceituais e estimular suas potencialidades e perspectivas para o cuidado de si. No caso do controle do peso, este processo pode contribuir para estimulá-lo a buscar conhecimentos voltados aos cuidados necessários, considerando que haverá maior conscientização e desenvolvimento de competências para uma tomada de decisões.

O pouco conhecimento sobre o processo de adoecimento pode ser um dos fatores que interferem na adesão ao tratamento e no controle metabólico e, considerando que uma pessoa com uma condição crônica necessita conviver com sua doença pelo resto da vida, as práticas educativas dão suporte para enfrentar e superar as intercorrências da doença e do tratamento. ${ }^{16}$

A subcategoria trocando e agregando informações revelou que o monitoramento remoto foi uma estratégia valiosa para esclarecer dúvidas, reforçar, relembrar e introduzir novos conhecimentos sobre o excesso de peso. Além disso, a subcategoria Sentindose satisfeita pelo cuidado revelou que a interação estabelecida com as mulheres durante o monitoramento trouxe motivação, incentivo e ofereceu força em momentos de desmotivação para o seguimento das metas pretendidas. $\mathrm{O}$ conhecimento $\mathrm{e}$ o acesso às informações são imprescindíveis para a realização de escolhas e o empoderamento para ação, constituindo-se em um elemento que agrega valor para o autocuidado. ${ }^{7}$

A subcategoria sentindo-se satisfeita pelo cuidado compartilhado revelou, também, que as orientações compartilhadas entre a enfermeira e as mulheres foram consideradas uma forma de expressão do cuidado em saúde, pois sentiram-se únicas e valorizadas ao ter alguém que, de modo autêntico e genuíno, preocupava-se com seu estado de saúde. A satisfação das participantes com o monitoramento representa um aspecto importante revelado neste estudo, pois é considerada uma das dimensões distintas da qualidade do cuidado e que repercute diretamente na adesão ao tratamento. ${ }^{19} \mathrm{Um}$ dos aspectos que contribui para a satisfação com a utilização da tecnologia da informação, como uma forma de cuidado, é a aceitação do usuário. ${ }^{12}$ Segundo este autor, a não conformidade pelo usuário pode causar insatisfação, independente de outros fatores.

Conforme preconizado, na interação com o usuário, o profissional deve olhar para além da doença obesidade e valorizar outros aspectos da saúde, como o bem-estar, a autoestima e a melhoria na qualidade de vida, ou seja, o foco de atenção não pode limitar-se à patologia e terapêutica, ${ }^{3}$ mas à pessoa com excesso de peso.

Ressalta-se que o sentimento de estar sendo cuidada foi contemplado na percepção das mulheres, pois o "sentir-se bem" e o "sentir-se cuidada" emergiu de várias falas. Estes são sentimentos importantes, considerando que a obesidade traz consigo estigmas, preconceitos e desvalorização, ${ }^{5}$ bem como, dificuldades inerentes à precariedade da assistência dos serviços de saúde. Portanto, agregar outros atributos ao cuidado com pessoas com excesso de peso é valioso.

Constatou-se que a vivência de um feedback interativo durante o monitoramento remoto de enfermagem ajudou as mulheres a promoverem mudanças concretas de comportamento, conforme expressou a categoria melhorando o cuidado de si e suas subcategorias. A correlação entre o conhecimento e a atitude no relato das mulheres, sugeriu que o aumento do conhecimento pode estar associado à predisposição para um melhor cuidado com a saúde, conforme evidenciado pelas subcategorias, mantendo-se na linha, incorporando e adaptando-se a novos comportamentos.

Várias mulheres descreveram mudanças de comportamento concretas advindas após as orientações compartilhadas durante o monitoramento remoto. As mulheres incorporaram novos comportamentos e adaptaram-se a novos hábitos, assim como, procuraram resistir às tentações que ameaçariam o controle do peso após as conquistas alcançadas. Estas mudanças e esforços foram, principalmente, relacionados à redução do comportamento sedentário e à melhora dos hábitos alimentares. $O$ aconselhamento de profissionais de saúde é efetivo para o direcionamento dos indivíduos à adoção de comportamento ativo. ${ }^{20}$ 
Para viver em uma sociedade moderna, a qual é extremamente obesogênica, é indispensável a inclusão de novos hábitos no cotidiano para o controle do peso, ${ }^{21}$ e as ações educativas se mostram efetivas, quando há uma participação e aceitação dos atores envolvidos, profissional de saúde e cliente.

Surgiu nas falas das mulheres, de forma frequente, a contribuição da intervenção como agente motivador para evitar a desistência do tratamento. Os depoimentos não foram casos isolados, mas recorrentes, o que leva à reflexão sobre a importância de contatos mais frequentes, assegurando a acessibilidade à enfermeira $e$ à troca de informações essenciais. Atividades educativas, além de propiciar compartilhamento de informações, esclarecimento de dúvidas, pode atenuar a ansiedade, preencher carências de sentir-se cuidado e fortalecer a autoestima.

A adoção de hábitos mais saudáveis de vida é necessária para o controle do peso e gera benefícios à saúde, e estes demandam comportamentos como motivação, disciplina e conhecimento. ${ }^{3}$ Estudo realizado sobre a adesão a modos saudáveis de vida, mediante aconselhamento por profissionais de saúde, observou a importância do aconselhamento como forma de auxiliar os indivíduos na busca pela melhoria da saúde, qualidade de vida e na adoção de modos saudáveis de vida. ${ }^{22}$

Relatos apontando que as ligações serviam de alerta, lembrete e estímulo para conservar-se firme quanto ao propósito de manter os hábitos necessários ao controle do peso conduzem à ideia de Freire, ${ }^{23}$ de que as palavras no processo de aprendizagem, medidas pela objetivação e pela imediatez da experiência, são interiorizadas e, posteriormente, passam por uma reflexão e crítica para novos projetos existenciais.

Para que ocorra a atitude de autocuidado, que conduz a hábitos de vida mais saudáveis, é necessária uma conscientização do indivíduo sobre sua condição de saúde e a relação desta com suas práticas de manutenção e recuperação da vida, do que, simplesmente, a oferta de uma prescrição profissional, ${ }^{3,24}$ pois, para haver a verdadeira aprendizagem, é necessário uma transformação real do sujeito por meio da construção e da reconstrução do saber ensinado. ${ }^{15}$ Assim, a percepção das mulheres relacionada ao benefício do monitoramento remoto, enquanto instrumento de despertar para conscientização para o autocuidado, permite ponderar que a forma como a comunicação foi feita considerouas sujeitos ativos de seu tratamento, durante todo o processo.

A percepção das participantes sobre o monitoramento remoto coadunou-se com o que vem sendo descrito na literatura, a qual aponta que programas com o uso de ferramentas ou tecnologias de comunicação na prestação dos cuidados de saúde podem ampliar e facilitar o acesso entre o profissional e o cliente. Outrossim, colaborar para a criação de vínculos, estimular a continuidade do tratamento, apoiar o processo de autocuidado e a participação ativa dos clientes na construção de conhecimentos e na tomada de decisão mais consciente sobre os cuidados a serem adotados para manutenção e recuperação da vida. ${ }^{9}$

Vale destacar que o papel dos profissionais, em sua prática de assistência à saúde de outras pessoas não se resume à mera aplicação de conhecimentos. Dessa forma, o monitoramento foi além de uma intervenção educativa, conseguindo alcançar necessidades, suprir carências com dimensões subjetivas e ajudar as mulheres a lograrem resultados favoráveis no controle do peso e satisfação com o monitoramento remoto. O modo como foi desenvolvido, com base numa abordagem individualizada e diferenciada, demostrando interesse em relação ao bem-estar das participantes, foi percebido de maneira positiva.

Embora, muitas vezes, as participantes tenham revelado que já haviam recebido as informações, as ligações telefônicas as ajudaram a obter resultados desejados no que se referia ao controle do peso. Além disso, ao considerar que as participantes do presente estudo eram acompanhadas por equipe multidisciplinar e os conhecimentos prévios se coadunavam com os conteúdos abordados na intervenção, mostrou-se que existe convergência teórica na abordagem entre os profissionais da equipe e os autores do projeto de monitoramento. A inexistência de divergência entre as orientações compartilhadas contribuiu para um relacionamento tranquilo entre os atores envolvidos no projeto, participantes e equipe de monitoramento e, por conseguinte, ampliou a formação de vínculos entre os mesmos.

No controle da obesidade, as medidas de apoio tornam mais factível à adesão às práticas saudáveis por indivíduos e coletividades, informados e motivados. ${ }^{17}$ Mudar o estilo de vida é complexo e os resultados nem sempre são animadores quando se trata de perder e manter o peso perdido. Muitos clientes recuperam o peso perdido e, por vezes, ainda adquirem mais alguns nos anos seguintes, sendo necessários programas de acompanhamento contínuos para evitar estas situações. Geralmente, os cuidados realizados por pessoas obesas vêm acompanhados de dificuldades em executá-los e, embora, muitas vezes, não consigam obter os resultados desejados, não deixaram de realizá-los, o que requer que tais esforços empenhados por esses indivíduos sejam valorizados pelos profissionais de saúde. ${ }^{24}$

Outro aspecto essencial para as participantes deste estudo diz respeito ao desejo de dar continuidade ao monitoramento remoto. É legítimo refletir sobre o atendimento dispensado a essas mulheres nesse processo, pois a obesidade, ao constituir uma doença de natureza complexa e multifatorial, requer abordagem centrada em intervenções para modificação do estilo de vida de forma contínua e frequente. O sucesso, em longo prazo, depende de constante vigilância na adequação dessas medidas, além de outros fatores, como apoio profissional, social, familiar e automonitorização. ${ }^{2,3}$ Desta forma, entende-se que cuidados mediados pelo monitoramento remoto podem criar maior vínculo profissional-cliente, pois este último pode participar, de forma ativa, das orientações, esclarecendo dúvidas e ampliando o acesso às informações relacionadas aos cuidados necessários ao controle do peso. ${ }^{12,13}$

\section{CONCLUSÕES E IMPLICAÇÕES PARA A PRÁTICA}

Esta pesquisa possibilitou conhecer a avaliação das mulheres com excesso de peso sobre o monitoramento remoto de enfermagem. A intervenção contribuiu para as mulheres 
aumentarem a consciência do cuidado de si, ao vivenciarem um feedback frequente e interativo com a enfermeira para o controle do peso, o qual propiciou trocar e agregar informações e a satisfação pelo cuidado recebido.

As interações vivenciadas com a enfermeira contribuíram para as mulheres melhorarem o cuidado de si, ou seja, para incorporarem e adaptarem-se a novos comportamentos fundamentais para o controle do peso. Perceberam resultados no controle do peso e desejaram a continuidade da intervenção, mostrando que houve satisfação com os resultados alcançados.

Vale ressaltar que, após a avaliação positiva da intervenção pelas mulheres, acredita-se que serviços ambulatoriais podem tornar-se um espaço privilegiado para o desenvolvimento de ações de incentivo e apoio à adoção de estilo de vida saudável, lançando mão de metodologias e estratégias inovadoras para ampliação do acesso e comunicação entre usuários e profissionais. O telemonitoramento representou uma ferramenta que oportunizou a continuidade e reforço ao cuidado e tratamento das mulheres com excesso de peso.

Por fim, destaca-se a importância de se conhecer a avaliação das participantes de um projeto dessa natureza, considerando que o mesmo pode ser usado por enfermeiros com os demais profissionais da equipe multidisciplinar, no acompanhamento e seguimento de pessoas com excesso de peso. Este conhecimento permite a adequação de novos projetos de intervenção educativa por meio de monitoramento remoto de enfermagem.

\section{FINANCIAMENTO}

Bolsa de Iniciação Científica do Programa de Bolsa de Iniciação Cientifica (PIBIC) da Fundação de Amparo a Pesquisa do Estado da Bahia (FAPESB) concedida a Giulia Araújo Ramos, 2016

Conselho Nacional de Desenvolvimento Científico e Tecnológico (CNPq), Brasil, Processo nº 421599/2016-2, projeto coordenado pela Dra. Fernanda Carneiro Mussi

\section{CONTRIBUIÇÃO DOS AUTORES}

Desenho do estudo. Catia Suely Palmeira. Giulia Araújo Ramos. Fernanda Carneiro Mussi.

Aquisição, análise de dados e interpretação dos resultados. Catia Suely Palmeira. Giulia Araújo Ramos. Fernanda Carneiro Mussi.

Redação e revisão crítica do manuscrito. Catia Suely Palmeira. Giulia Araújo Ramos. Fernanda Carneiro Mussi.

Aprovação da versão final do artigo. Responsabilidade por todos os aspectos do conteúdo e a integridade do artigo publicado. Catia Suely Palmeira. Giulia Araújo Ramos. Fernanda Carneiro Mussi.

\section{EDITOR ASSOCIADO}

Ana Luiza de Oliveira Carvalho

\section{REFERÊNCIAS}

1. World Health Organization. Obesity and overweight [Internet]. Geneva: WHO; 2017 [citado 2017 mar 23]. Disponível em: http://www.who.int/ mediacentre/factsheets/fs311/en/

2. Associação Brasileira para o Estudo da Obesidade e da Síndrome Metabólica. Diretrizes brasileiras de obesidade. $4^{a}$ ed. São Paulo: ABESO; 2016 [citado 2017 mar 23]. Disponível em: http://www.abeso. org.br/diretrizes

3. Brasil. Ministério da Saúde. Secretaria de Atenção à Saúde. Departamento de Atenção Básica. Estratégias para o cuidado da pessoa com doença crônica: obesidade [Internet]. Brasília: Ministério da Saúde; 2014.212 p. [citado 2017 mar 23]. Disponível em: https://crn5.org.br/estrategiaspara-o-cuidado-da-pessoa-com-doenca-cronica-obesidade/

4. Goettler A, Grosse A, Sonntag D. Productivity loss due to overweight and obesity: a systematic review of indirect costs. BMJ Open. 2017;7(10):e014632. http://dx.doi.org/10.1136/bmjopen-2016-014632. PMid:28982806.

5. Macedo TTS, Portela PP, Palmeira CS, Mussi FC. Percepção de pessoas obesas sobre o seu corpo. Esc Anna Nery. 2015;19(3):505-10. http:// dx.doi.org/10.5935/1414-8145.20150067.

6. Falkenberg MB, Mendes TPL, Moraes EP, Souza EM. Educação em saúde e educação na saúde: conceitos e implicações para a saúde coletiva. Cien Saude Colet. 2014;19(3):847-52. http://dx.doi.org/10.1590/141381232014193.01572013. PMid:24714898.

7. Taddeo PS, Gomes KWL, Caprara A, Gomes AMA, Oliveira GC, Moreira TMM. Acesso, prática educativa e empoderamento de pacientes com doenças crônicas. Cien Saude Colet. 2012;17(11):2923-30. http:// dx.doi.org/10.1590/S1413-81232012001100009.

8. Godoy SCB, Guimarães EMP, Assis DSS. Avaliação da capacitação dos enfermeiros em unidades básicas de saúde por meio da telenfermagem. Esc Anna Nery. 2014;18(1):148-55. http://dx.doi.org/10.5935/14148145.20140022.

9. Mussi FC, Palmeira CS, Silva RM, Costa ALS. Telenfermagem: contribuições para o cuidado em saúde e a promoção do conforto. Rev Cient Sena Aires. [Internet]. 2018; [cited 2019 mar 2];7(2):76-9. Disponível em: http://revistafacesa.senaaires.com.br/index.php/revisa/ issue/view/18

10. Brahmbhatt $\mathrm{DH}$, Cowie MR. Remote management of heart failure: an overview of telemonitoring technologies. Card Fail Rev. 2019;5(2):8692. http://dx.doi.org/10.15420/cfr.2019.5.3. PMid:31179018.

11. Hernandez-Quiles C, Bernabeu-Wittel M, Garcia-Serrano MDR, Vergara-Lopez S, Perez-de-Leon JA, Ruiz-Cantero A et al. A multicenter randomized clinical trial to evaluate the efficacy of telemonitoring in patients with advanced heart and lung chronic failure. Study protocol for the ATLAN_TIC project. Contemp Clin Trials Commun. 2020;17:100512. http://dx.doi.org/10.1016/j.conctc.2019.100512. PMid:31938754.

12. Shannon HH, Joseph R, Puro N, Darrell E. Use of technology in the management of obesity: a literature review. Perspect Health Inf Manag. 2019;16(Fall):1c. PMid:31908626.

13. Palmeira CS, Mussi FC, Ramos GA, Jesus NV, Macedo TTS, Dal Sasso GTM. Nursing protocol for remote monitoring of women with excessive weight. Texto Contexto Enferm. 2019;28:e20170400. http:// dx.doi.org/10.1590/1980-265x-tce-2017-0400.

14. Minayo MCS, Deslandes SF. Pesquisa social: teoria, método e criatividade.

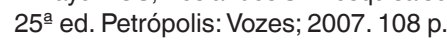

15. Vale EG, Pagliuca LMF. Construction of a nursing care concept: contribution for undergraduate nursing education. Rev Bras Enferm. 2011;64(1):106-13. http://dx.doi.org/10.1590/S0034-71672011000100016. PMid:21468497.

16. Rodrigues FFL, Santos MA, Teixeira CRS, Gonela JT, Zanetti ML. Relationship between knowledge, attitude, education and duration of disease in individuals with diabetes mellitus. Acta Paul Enferm. 2012;25(2):284-90. http://dx.doi.org/10.1590/S0103-21002012000200020.

17. Burgess E, Hassmén P, Pumpa KL. Determinants of adherence to lifestyle intervention in adults with obesity: a systematic review. Clin Obes. 2017;7(3):123-35. http://dx.doi.org/10.1111/cob.12183. PMid:28296261.

18. Freire P. Pedagogia da autonomia. São Paulo: Paz e Terra, 2011. 
19. Marinho NBP, Freitas RWJF, Lisboa KWSC, Alencar AMPG, Rebouças VCF, Damasceno MMC. Evaluation of the satisfaction of users of a service specialized in diabetes mellitus. Rev Bras Enferm. 2018;71(Suppl 1):599606. http://dx.doi.org/10.1590/0034-7167-2017-0554.PMid:29562017.

20. Häfele V, Siqueira F. Aconselhamento para atividade física e mudança de comportamento em Unidades Básicas de Saúde. Rev Bras Ativ Fís Saúde. 2017;21:581-92. http://dx.doi.org/10.12820/ rbafs.v.21n6p581-592.

21. Bevilaqua CA, Pelloso SM, Marcon SS. Stages of change of behavior in women on a multi-professional program for treatment of obesity. Rev
Lat Am Enfermagem. 2016;24:e2809. http://dx.doi.org/10.1590/15188345.0549.2809. PMid:27737377.

22. Toledo MTT, Abreu MN, Lopes ACS. Adesão a modo saudáveis de vida mediante aconselhamento por profissionais de saúde. Rev Saude Publica. 2013;47(3):540-8. http://dx.doi.org/10.1590/S00348910.2013047003936. PMid:24346567.

23. Freire P. Pedagogia do oprimido. Rio de Janeiro: Paz e Terra; 2010.

24. Araujo FM, González AD, Silva LC, Garanhani ML. Obesidade: possibilidades de existir e práticas de cuidado. Saude Soc. 2019;28(2):249-60. http:// dx.doi.org/10.1590/s0104-12902019170152. 